\title{
Effectiveness and Estimation of Cost-Effectiveness of a Group-Based Multicomponent Physical Exercise Programme on Risk of Falling and Frailty in Community-Dwelling Older Adults
}

\author{
Tamara Alhambra-Borrás ${ }^{1, *}$, Estrella Durá-Ferrandis ${ }^{1}$ and Maite Ferrando-García ${ }^{2}$ \\ 1 Polibienestar Research Institute, Universitat de València, 46022 Valencia, Spain; estrella.dura@uv.es \\ 2 Kveloce I+D+i, R\&D+i Consultancy, 46002 Valencia, Spain; mferrando@kveloce.com \\ * Correspondence: tamara.alhambra@uv.es
}

Received: 17 April 2019; Accepted: 11 June 2019; Published: 13 June 2019

\begin{abstract}
This study analyses the effectiveness and cost-effectiveness of a group-based multicomponent physical exercise programme aimed at reducing the risk of falling and frailty in community-dwelling older adults. This is a pretest-posttest non-equivalent control group design, with an intervention group and a comparison group. Participants were evaluated at baseline and after 9 months. The effectiveness analyses showed significant reduction in the risk of falling $(-45.5 \%$; $p=0.000)$ and frailty $(-31 \% ; p=0.000)$ after the intervention for the participants in the physical exercise programme. Moreover, these participants showed an improvement in limitations in activities of daily living, self-care ability and the use of health resources, physical performance, balance and body mass index. The cost-effectiveness analyses showed that the intervention was cost-saving and more effective than usual care scenario. A novel group-based multicomponent physical exercise programme showed to be more effective and cost-effective than usual care for older adults suffering from risk of falling and frailty.
\end{abstract}

Keywords: risk of falling; frailty; falls; older adults; physical exercise; ageing

\section{Introduction}

Falls and frailty are crucial health issues affecting older adults. Both share many significant domains and represent a major burden on the healthcare system.

Falls represent an important and complex health problem because of its high prevalence among older adults and its consequences at physical, psychological and social level [1]. One in three people over 65 falls at least once a year worldwide [2]. According to the European Injury Data Base [3], among older adults $58 \%$ of emergency departments attendance in hospitals are due to falls; 2.3 million people over 65 are estimated to be treated every year in an emergency department because of a fall, whereof approximately 1.5 million are admitted to the hospital. The health care expenditure for treating fall-related injuries in the EU is estimated to be 25 billion euros each year.

On the other hand, frailty is the most problematic expression of population ageing. Traditionally, frailty has been defined as a medical syndrome consisting of solely physical components-frailty phenotype framework [4]. However, in the last decade, a multidimensional integral perspective of frailty has been introduced defining frailty as "a dynamic state affecting an individual who experiences losses in one or more domains of human functioning (physical, psychological, social) that are caused by the influence of a range of variables and which increases the risk of adverse outcomes." [5]. Moreover, frailty can be seen as a relative state that may change over time [6,7]. Although frailty is a complex condition which increases with age, no consensus exists about the prevalence rates of frailty. This is 
partly due to the operational definition of frailty used. Among those studies that used the physical phenotype, prevalence varied from $4 \%$ to $17 \%$; while in the studies that measured frailty using comprehensive definitions, frailty prevalence ranged from $4.2 \%$ to $59.1 \%$.

Both falls and frailty have a proven association with mortality, morbidity, hospitalisations, reduced mobility, limitations in activities of daily living (ADLs), disability, fear of falling, a reduced self-care ability, as well as social isolation and a reduced quality of life [8-12]. Furthermore, falls and frailty and their consequences have proven to increase health care utilisation and expenditures $[13,14]$.

The high prevalence and the consequences on health of the risk of falling and frailty among older people highlight the need to develop and to implement strategies that effectively address these conditions. In this regard, the regular practise of physical activity has proved to prevent and reduce frailty and the risk of falling, even the injuries resulting from falls $[15,16]$. Balance and strength training have showed to be the most effective kinds of training $[17,18]$. In this regard, the OTAGO programme [19] has been identified as one of the most effective multicomponent programmes [20,21].

In this study, the purpose was to analyse the effect of a group-based multicomponent physical exercise programme on the reduction of the risk of falling and frailty in community-dwelling older adults. This programme offers a modified format of OTAGO programme including evidence-based improvements. On the one hand, 26 additional exercises to the 34 existing ones are included in the multicomponent programme. The additional exercises were designed by a physiotherapist and included dynamic self-resistance exercises. These kinds of exercises are designed to harmoniously develop the muscles of the body without using any equipment but using the body as a resistance. Muscle strength, as well as balance, play a central role in fall and frailty prevention and their consequences $[17,18]$. On the other hand, as part of OTAGO, a walking plan of two 30-minute walks per week is included. However, our programme does not include walking prescription because several systematic reviews of the literature have pointed out that programmes that do not include walking plans as part of their intervention are more effective on risk of falling than those including walking plans $[17,18]$. Moreover, our programme, instead of being individually prescribed and delivered at participant's home, offers a group-based exercise programme, which has previously shown to motivate participants to perform exercises more effectively [22,23]. Finally, the vast majority of studies using the OTAGO programme have been focused on reducing falls and related injuries, and our study also explores as a primary outcome the impact on frailty.

Furthermore, this study analysed the impact of the intervention on other health-related variables, namely limitations in ADLs, health-related quality of life, self-care agency, self-efficacy related to falls and the use of health resources; being the hypothesis that after intervention, participants would also show an improvement in these variables. In addition, a secondary objective was to estimate the cost-effectiveness of the intervention in order to know if our physical exercise programme was potentially cost-saving in comparison to usual care for older people who were frail or at risk of falling.

\section{Materials and Methods}

\subsection{Study Design}

This prospective longitudinal study followed a quasi-experimental design, concretely a pretest-posttest non-equivalent control group design, with an intervention group and a comparison group, where participants were not randomly assigned to the two groups. Participants included in this study were evaluated at baseline and after 9 months.

All participants included in the study provided written informed consent. The design and informed consent procedure of the study were approved by the Ethics Committee of the Consorcio Hospital General Universitario de Valencia (Spain, 20131201). 


\subsection{Study Participants and Inclusion Criteria}

The study population consisted of community-dwelling people aged 65 years or older from the city of Valencia (Spain) with no severe physical or cognitive limitations. These two exclusion criteria were assessed by general practitioners from both healthcare centres participating in the study.

From February 2015 to July 2015 a total of 500 participants from two healthcare centres in the city of Valencia were invited to participate in the study. Each of these healthcare centres corresponded at convenience to one of the study groups: intervention $(N=258)$ and comparison group $(N=242)$.

Participants were screened for risk of falling and/or frailty by individual assessments carried out at each participant's home by previous appointment and after accepting to participate in the study at the healthcare centre. No statistically significant differences were found in any of the relevant demographic and health-related variables between the intervention group and the comparison group. After this baseline assessment those participants from both healthcare centres meeting the inclusion criteria (having risk of falling and/or frailty) were included in the study. Among participants from healthcare centre 2 (where the intervention was conducted), acceptance to participate in the physical exercise programme was also established as an inclusion criterion. Therefore, subjects from healthcare centre 2 had to agree to enrol into the intervention prior to be included as part of the intervention group, being this one of the main reasons why the intervention group sample was reduced from 258 to 74 .

A total of 175 participants composed the comparison group and 74 participants composed the intervention group (Figure 1).

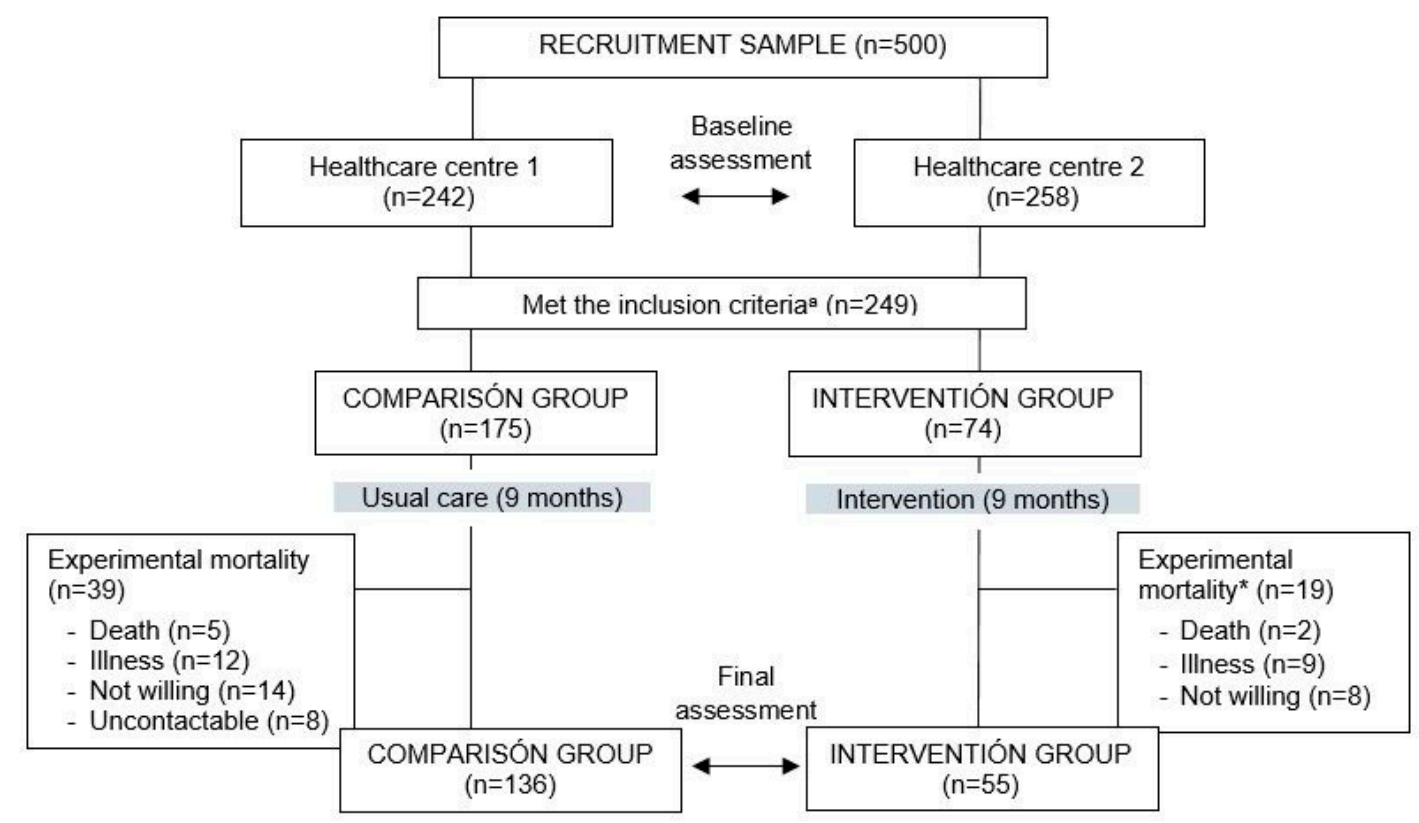

Figure 1. Flow of participants through the study. ${ }^{a}$ Inclusion criteria: Having risk of falling and/or frailty and, for intervention group, acceptance to participate in the physical exercise programme. * Experimental mortality includes the participants who dropped out of the study after having met the inclusion criteria.

\subsection{Outcome Measures}

\subsubsection{Primary Outcomes}

Risk of falling was assessed among participants using the following criteria recommended by the American Geriatrics Society \& British Geriatrics Society [24]: (1) had fallen at least twice during the previous 12 months; (2) had self-reported walking problems; (3) had self-reported balance problems (criteria 2 and 3 were assessed using questions 3 and 4 of the TFI [6]). Moreover, a fourth criterion on 
having a fear of falling [25] was also included, as suggested by several studies [26,27]. Participants were classified as having risk of falling if they answered yes on criterion 1 or yes on criterion 4 plus yes on one or both criteria 2 and 3 . The responses obtained from these questions were dichotomised to yes or no for risk of falling.

Frailty was assessed through the Tilburg Frailty Index (TFI) [6]. The TFI's total score ranges from 0 to 15 . The higher the score, the higher one's frailty. Frailty is diagnosed with a TFI score $\geq 5$. The TFI is based on a multidimensional approach to frailty, including physical, psychological and social aspects, and it showed a robust evidence of reliability and validity [28]. Cronbach's alpha was 0.69 for the participants in this study.

\subsubsection{Secondary Outcomes}

Limitations in activities of daily living were measured using the Groningen Activity Restriction Scale (GARS) [29]. Cronbach's alpha for the study participants was 0.91. Self-care agency, as the individual's ability to evaluate health-related needs and conduct self-care activities aimed at promoting and maintaining health and well-being [30], was evaluated using the Spanish version of the Appraisal of Self-care Agency Scale-Revised (ASA-R) [31] and Cronbach's alpha was 0.77 for the respondents. Besides, participants' self-efficacy related to falls was measured through the Falls Efficacy Scale-International (FES-I) [32], which Cronbach's alpha was 0.83 for the study participants. This questionnaire is intended to assess fear of falling among older people. Fear of falling may cause the reduction of activities, leading to a reduced physical activity and mobility, which paradoxically increase the risk of falls and injury [33,34]. Participants' quality of life was measured using the SF-12 Health Survey [35]. SF-12 Cronbach's alpha for the study participants was 0.90. The use of healthcare resources was also evaluated at baseline and after 9 months, asking participants an estimation of all doctor visits and hospitalisations over the previous 12 months (at baseline) or the previous 9 months (at post-intervention).

Furthermore, the intervention group participants-those included in the physical exercise programme-were also assessed through two extra measures: physical performance and body composition. Physical performance was evaluated using the Short Physical Performance Battery (SPPB) [36] which includes the balance test, the gait speed test and the chair stand test. Finally, body composition, including weight, body mass index (BMI), body fat ( $\mathrm{kg}$ ), lean body mass $(\mathrm{kg})$ and body water (kg), was measured using a Tanita scale (TBF300), a measure of bioelectrical impedance.

Finally, for the cost-effectiveness analyses of the intervention the following costs were estimated: the cost related to the physical exercise programme (costs of the physiotherapist in charge of the programme implementation and supervision) and the healthcare costs related to the use of healthcare resources (number of doctor's visits and number of hospitalisations in both intervention and comparison groups). Furthermore, the quality of life, which was measured using the SF-12 Health Survey, was also used for these analyses.

\subsection{Intervention}

Participants allocated in the intervention group enrolled in a 9 months physical exercise programme. During this period, participants met twice a week in sessions of $45 \mathrm{~min}$. In each session an exercise routine was performed and supervised by a physiotherapist. All intervention sessions were conducted in a community centre located in the participants neighbourhood.

The physical exercise programme was a multicomponent intervention including both balance and strength training, which have showed to be the most effective kind of training in preventing falls and frailty $[2,18,26,37,38]$. A total of 60 exercises conformed the programme, from which 34 exercises were based on the OTAGO programme [19], one of the most successful programmes for preventing falls [21], and the other 26 exercises were designed ad hoc by a physiotherapist. The ad hoc exercises include dynamic self-resistance training, which is a specific strength training that involves flexing the muscles hard while also moving. The programme comprises a total of ten routines with 6 exercises each one. It 
is important to highlight that the OTAGO programme was originally designed to be implemented individually at home, while we adapted it to be delivered in group sessions. Physical exercise in a group format has proven to be as effective, or even more effective, than individual sessions [22,23]. A detailed description of the physical exercise programme implemented in this study has been included as Supplementary Materials S1.

\subsection{Data Analyses}

Before the effectiveness analyses, we conducted analyses on the experimental mortality in order to know if people who dropped out differ significantly from participants that stayed in. These analyses were conducted for the intervention variables (risk of falling and frailty) using contrast test Chi-square tests for the risk of falling and t-test for frailty.

The effectiveness analysis of the intervention was conducted through the analyses of the risk of falling and frailty. Intragroup analyses were performed to know the evolution of these variables in the comparison and the intervention group, and intergroup analyses to compare the two groups in two different moments: pre-intervention and 9 months later.

Given the categorical nature of the risk of falling, intergroup analyses were performed using Chi-square test, and intragroup differences were analysed using McNemar test. As frailty was a continuous variable a Repeated Measures ANOVA test was selected to analyse intragroup and intergroup differences. However, this analysis showed that the variable frailty did not meet the homoscedasticity criterion. Thus a transformation of the variable using Box-Cox power transformation was carried out. However, after an exponential transformation, the frailty variable still did not meet the homoscedasticity criterion. So, non-parametric test was selected. In particular, Wilcoxon's signed rank test was used to calculate intragroup differences and Mann-Whitney $U$ test for intergroup differences.

The impact of the intervention on other health-related variables-physical performance, body composition, limitations in ADLs, self-care agency, self-efficacy related to falls, quality of life and use of healthcare resources-was also measured using McNemar test and the Wilcoxon test, as these variables did not meet the assumptions of the parametric test.

Finally, the potential cost-effectiveness of the intervention was estimated by means of the Monitoring and Assessment Framework for the European Innovation Partnership on Active and Healthy Ageing (MAFEIP) tool [39]. Concretely, incremental costs and effects analyses, and cost-effectiveness analyses were carried out using this framework, which provides a web application enabling cost-effectiveness estimations based on the principles of Decision Analytic Modelling (DAM) and the traditional Markov model to assess the impact of innovations on health outcomes and resource use [39]. To provide the input required by MAFEIP, the intervention and usual care costs, and the utility (quality of life weight) were estimated. The central element in the cost-estimation were the human resources expenses (physiotherapist) required for the programme implementation, which was calculated to be 1560 euros $(1.5 \mathrm{~h} /$ week $\times 52$ weeks $\times 20$ euros/h) for an average of 15 participants attending the programme sessions, which results in an intervention cost per person of $104 €$. On the other hand, the costs related to the use of healthcare resources (frequency of doctor's visits and hospitalisation) during the study ( 9 months) was also estimated for both intervention and comparison groups. In order to calculate the costs of doctors' visits, an average rate of $29.23 €$ per hour was assumed (based on the national statistics on social security for this professional category). The costs estimation per hospital bed-day was $733.56 €$ (calculated from the data on the hospital inpatient curative care expenditure and the number of hospital curative care bed-days; both variables available in Eurostat for Spain). With these assumptions, health resources consumption costs were estimated per group at two states, baseline and deteriorated, using MAFEIP. Healthcare costs for patients in baseline state were estimated to be $1615.02 €$ for the intervention group and $1630.22 €$ for the comparison group; while for those in deteriorated state were $3130.96 €$ for intervention group and $9030.13 €$ for the comparison group. To calculate the effects on health-related quality of life, the EQ-5D is recommended to be used within the MAFEIP context. However, this study did not use this questionnaire, instead the SF-12 
Health Survey was used [35]. Thus, SF-12 scores were converted into EQ-5D scores using an algorithm developed by Gray, Rivero-Arias and Clarke [40].

\section{Results}

The age of the participants ranged from 65 to 92 years old and it included both females $(73.2 \%)$ and males (26.8\%). Intervention group participants were slightly younger than comparison group participants (mean age 76.7 vs. 78.4) and the percentage of women was higher in the intervention group ( $81.8 \%$ vs. $64.7 \%)$. Among those who dropped out (experimental mortality), no statistically significant differences were found in risk of falling and frailty neither for the intervention group (risk of falling $p=0.85$; frailty $p=0.08$ ) nor for the comparison group (risk of falling $p=0.63$; frailty $p=0.55$ ). The exercise group mean attendance throughout the study was $85 \%$ of the planned sessions. A session was considered successfully finished when all exercises were completed.

After attending the physical exercise programme intervention group participants presented a significant reduction in both the risk of falling and frailty. The risk of falling was reduced by $45.5 \%$ $(p=0.000)$ among the older people who attended the intervention. On the other hand, in the same time period, comparison group participants suffered a slight increase in falls risk, but it was not statistically significant $(p=1.000)$. Moreover, intergroup differences were significant before and after the intervention. In fact, the intervention participants showed higher prevalence of risk of falling at baseline, while after the intervention period the comparison group presented higher scores on falls risk (Table 1). In the case of frailty, similar results as for the risk of falling were found. The intervention demonstrated a significant reduction of frailty of $31 \%(p=0.000)$ among intervention group participants, while the comparison group did not show a statistically significant reduction in frailty $(p=1.000)$. Furthermore, intergroup differences also showed a reverse trend before and after intervention in both groups (Table 2).

Table 1. Intragroup and intergroup differences of risk of falling *.

\begin{tabular}{cccc}
\hline Risk of Falling Differences & Intervention Group & Comparison Group & Intergroup Differences \\
\hline PRE $\boldsymbol{n}(\%)$ & $50(90.9 \%)$ & $86(63.2 \%)$ & $\mathrm{X}^{2}=14.628 ; p=0.000$ \\
POST $\boldsymbol{n} \mathbf{( \% )}$ & $25(45.4 \%)$ & $87(64.0 \%)$ & $\mathrm{X}^{2}=5.536 ; p=0.019$ \\
\% of change & $-45.5 \%$ & $+0.8 \%$ & \\
Intragroup differences & McNemar; $p=0.000$ & McNemar; $p=1.000$ & \\
Effect size $^{* *}$ & 0.29 & 0.38 & \\
\hline
\end{tabular}

${ }^{*}$ Intragroup differences were analysed using McNemar test and intergroup analyses using Chi-square test. ${ }^{* *}$ Effect size effect was calculated using Cramer's V (small effect $=0.2$, medium effect $=0.5$, large effect $=0.8$ ).

Table 2. Intragroup and intergroup differences of frailty *.

\begin{tabular}{|c|c|c|c|}
\hline Frailty Differences & Intervention Group & Comparison Group & Intergroup Differences \\
\hline PRE Mean \pm SD & $6.20 \pm 3.15$ & $5.21 \pm 2.24$ & $\begin{array}{c}\mathrm{U} \text { de Mann-Whitney }=3031.5 \\
p=0.039\end{array}$ \\
\hline POST Mean \pm SD & $4.27 \pm 2.69$ & $5.01 \pm 2.26$ & $\begin{array}{c}\mathrm{U} \text { de Mann-Whitney }=3054.5 ; \\
p=0.046\end{array}$ \\
\hline $\begin{array}{l}\% \text { of change } \\
\text { Intragroup differences } \\
\text { Effect size }\end{array}$ & $\begin{array}{c}-31 \% \\
\text { Wilcoxon } \mathrm{z}=-4.373 ; p=0.000 \\
0.59\end{array}$ & $\begin{array}{c}-4 \% \\
\text { Wilcoxon } \mathrm{z}=-1.142 ; p=0.253 \\
0.09\end{array}$ & \\
\hline
\end{tabular}

Physical performance and body composition were assessed only among intervention group participants (Table 3). Participants in the physical exercise programme showed an improvement in all the variables measured through the Short Physical Performance Battery. This improvement was found statistically significant for the overall scale $(p=0.009)$, which measures physical performance, and for 
the balance test $(p=0.009)$. The impact of the physical exercise programme on the body composition was moderated, even though all the body-related variables were slightly improved. This improvement was only found statistically significant for the body mass index (BMI), which showed a reduction of 0.4 points after intervention $(p=0.45)$.

Table 3. Intragroup differences (intervention group only) of physical performance and body composition *.

\begin{tabular}{lcccc}
\hline $\begin{array}{c}\text { Physical Performance and Body } \\
\text { Composition Variables }\end{array}$ & $\begin{array}{c}\text { PRE } \\
\text { Mean } \pm \text { SD }\end{array}$ & $\begin{array}{c}\text { POST } \\
\text { Mean } \pm \text { SD }\end{array}$ & $\begin{array}{c}\text { Pre-Post } \\
\text { Difference }\end{array}$ & Effect Size ** \\
\hline $\begin{array}{l}\text { Physical Performance (SPPB) } \\
\text { SPPB total }\end{array}$ & $7.71 \pm 2.07$ & $8.35 \pm 2.15$ & $p=0.009$ & 0.35 \\
$\quad$ Balance test & $3.18 \pm 0.98$ & $3.51 \pm 0.84$ & $p=0.009$ & 0.35 \\
$\quad$ Gait speed test & $2.44 \pm 1.03$ & $2.62 \pm 1.01$ & $p=0.176$ & 0.18 \\
$\quad$ Chair stand test & $2.09 \pm 1.06$ & $2.22 \pm 1.17$ & $p=0.225$ & 0.16 \\
\hline Body composition (Tanita scale) & & & & 0.07 \\
$\quad$ Weight & $70.95 \pm 14.02$ & $70.39 \pm 13.9$ & $p=0.152$ & 0.07 \\
$\quad$ BMI & $30.96 \pm 6.07$ & $30.55 \pm 5.95$ & $p=0.045$ & 0.04 \\
$\quad$ Body fat (kg) & $27.61 \pm 10.07$ & $26.95 \pm 9.87$ & $p=0.075$ & 0.01 \\
$\quad$ Lean body mass (kg) & $43.34 \pm 6.66$ & $42.72 \pm 8.52$ & $p=0.930$ & 0.02 \\
$\quad$ Body water (kg) & $31.95 \pm 4.78$ & $31.82 \pm 4.95$ & $p=0.868$ & \\
\hline
\end{tabular}

* Intragroup differences were analysed using T-test and Wilcoxon's signed rank test. ${ }^{* *}$ Effect size was calculated using Cohen's $d$ for Weight, BMI and Body fat (small effect $=0.2$, medium effect $=0.5$, large effect $=0.8$ ); the effect size for the other variables was calculated using $\mathrm{r}=\mathrm{Z} / \sqrt{ } \mathrm{N}$ (r: effect size; $\mathrm{Z}$ : $\mathrm{Z}$ value from Wilcoxon test; $\mathrm{N}$ : Observation number; small effect $=0.2$, medium effect $=0.5$, large effect $=0.8$ ). SPPB: Short Physical Performance Battery.

The impact of the intervention on the health-related variables measured in this study was moderated (Table 4). Intervention group participants improved slightly but not significantly the quality of life (SF-12 Physical Health $p=0.200$; SF-12 Mental Health $p=0.535$ ) compared to the comparison group who presented a significant worsening of the quality of life after 9 months (SF-12 Physical Health $p=0.001$; SF-12 Mental Health $p=0.003$ ). Both groups presented a better ability for self-caring after the intervention period (intervention group $p=0.001$; comparison group $p=0.000$ ). The intervention group also presented a significant improvement in the limitation in ADLs, which were reduced by $6 \%(p=0.011)$ after the intervention, while the comparison group experienced an increase of these limitations over the same period, but it was not statistically significant $(p=0.905)$. The average number of doctor visits was significantly reduced among intervention group participants $(p=0.000)$. Prior to be included in the intervention, this group visited the doctor an average of 8.8 times per year, while after the intervention this average was reduced to 5.9 visits per year. Hospitalisation was also reduced by $50 \%(p=0.508)$ among intervention group participants and increased by $14 \%(p=0.832)$ in the comparison group, but these numbers were not found statistically significant. 
Table 4. Intragroup differences of health-related variables *.

\begin{tabular}{|c|c|c|c|}
\hline \multicolumn{2}{|c|}{ Health-Related Variables } & \multirow{4}{*}{$\begin{array}{c}\text { Intervention Group } \\
+7 \% \\
p=0.200 \\
0.17\end{array}$} & \multirow{4}{*}{$\begin{array}{c}\text { Comparison Group } \\
-9 \% \\
p=0.001 \\
0.28\end{array}$} \\
\hline SF-12 Phycical Health & $\%$ of change & & \\
\hline SF-12 Pnysical Healtn & Intragroup difference & & \\
\hline & Effect size ${ }^{* *}$ & & \\
\hline \multirow{3}{*}{ SF-12 Mental Health } & $\%$ of change & $-2 \%$ & $-7 \%$ \\
\hline & intragroup difference & $p=0.535$ & $p=0.003$ \\
\hline & Effect size $e^{* *}$ & 0.08 & 0.25 \\
\hline \multirow{3}{*}{ Self-care agency } & $\%$ of change & $+7 \%$ & $+8 \%$ \\
\hline & Intragroup difference & $p=0.001$ & $p=0.000$ \\
\hline & Effect size ${ }^{* *}$ & 0.48 & 0.42 \\
\hline \multirow{3}{*}{ Limitation in ADLs } & $\%$ of change & $-6 \%$ & $+3 \%$ \\
\hline & Intragroup difference & $p=0.011$ & $p=0.905$ \\
\hline & Effect size ${ }^{* *}$ & 0.35 & 0.01 \\
\hline \multirow{3}{*}{ Falls self-efficacy } & $\%$ of change & $-9 \%$ & $+4 \%$ \\
\hline & Intragroup difference & $p=0.098$ & $p=0.298$ \\
\hline & Effect size ${ }^{* *}$ & 0.22 & 0.09 \\
\hline \multirow{3}{*}{ Average doctor visits } & $\%$ of change & $-33 \%$ & $-7 \%$ \\
\hline & Intragroup difference & $p=0.000$ & $p=0.070$ \\
\hline & Effect size $* *$ & 0.48 & 0.15 \\
\hline \multirow{3}{*}{ Hospitalisation } & $\%$ of change & $-50 \%$ & $+14 \%$ \\
\hline & Intragroup difference & $p=0.508$ & $p=0.832$ \\
\hline & Effect size ${ }^{* *}$ & 0.08 & 0.18 \\
\hline
\end{tabular}

* Intragroup differences were analysed using Wilcoxon's signed rank test and McNemar test. ${ }^{* *}$ Effect size were calculated using $\mathrm{r}=\mathrm{Z} / \sqrt{ } \mathrm{N}$ ( $\mathrm{r}$ : effect size; $\mathrm{Z}$ : $\mathrm{z}$ value from Wilcoxon test; $\mathrm{N}$ : Observation number; $\mathrm{r}$ can be interpreted as small effect $=0.1$, medium effect $=0.3$, large effect $=0.5$ ). The effect size of hospitalisation was calculated using phi from McNemar test (Phi can be interpreted as small effect $=0.1$, medium effect $=0.3$, large effect $=0.5$ ). ADLS: activities of daily living.

Finally, cost-effectiveness analyses of the intervention were conducted using the MAFEIP tool. The previous results on the intervention effect were introduced together with the costs assumptions in the web-based tool to provide utility estimations for the two different health status as recommended by the framework; this is baseline health status and deteriorated health status [39]. Table 5 shows the cost and utility values used in the model.

Table 5. Input data used to populate the Monitoring and Assessment Framework for the European Innovation Partnership on Active and Healthy Ageing (MAFEIP) model.

\begin{tabular}{lcc}
\hline \multicolumn{1}{c}{ Variables Used in MAFEIP } & Intervention Group & Comparison Group \\
\hline Costs & & \\
Recurring cost per patient/year & $104 €$ & - \\
(intervention) & $1615.02 €$ & $1630.22 €$ \\
Healthcare cost-baseline & $3130.96 €$ & $9030.13 €$ \\
Healthcare cost-deteriorated & & \\
\hline Utility ** & 0.81 & 0.81 \\
Baseline state * & 0.75 & 0.75 \\
Deteriorated state * & & \\
\hline
\end{tabular}

* Baseline state includes those participants who show an improvement in the risk of falls and/or frailty at the final evaluation and Deteriorated state includes those participants showing a worsening in the risk of falling and/or frailty or those that show no progression (after 9 months remain the same). ${ }^{* *}$ Utility is the quality of life weight (utility of 1 would refer to the quality of life in perfect health and a utility of 0 would refer to no quality or dead).

Incremental costs analysis referred to the difference between the cost that a person from a specific age and gender would have if he/she received the intervention minus the cost that would have if 
he/she followed usual care. The results of this analysis showed that incremental costs by age were negative (Figure 2) meaning that, for older adults suffering from risk of falling and/or frailty, usual care was more expensive than the intervention. Moreover, the upward trend of the graphic meant that the older the participant the less savings. On the other hand, the incremental effects values referred to how much quality of life (utility) was gained when the intervention was used instead of the usual care. These were also showed per age-gender group. The results of the incremental effects by age were also positive, due to the fact that the physical exercise programme increased quality of life and health outcomes among participants (Figure 3). In this case, the graphic showed a downward trend meaning that the improvement was less noticeable as the age increased.

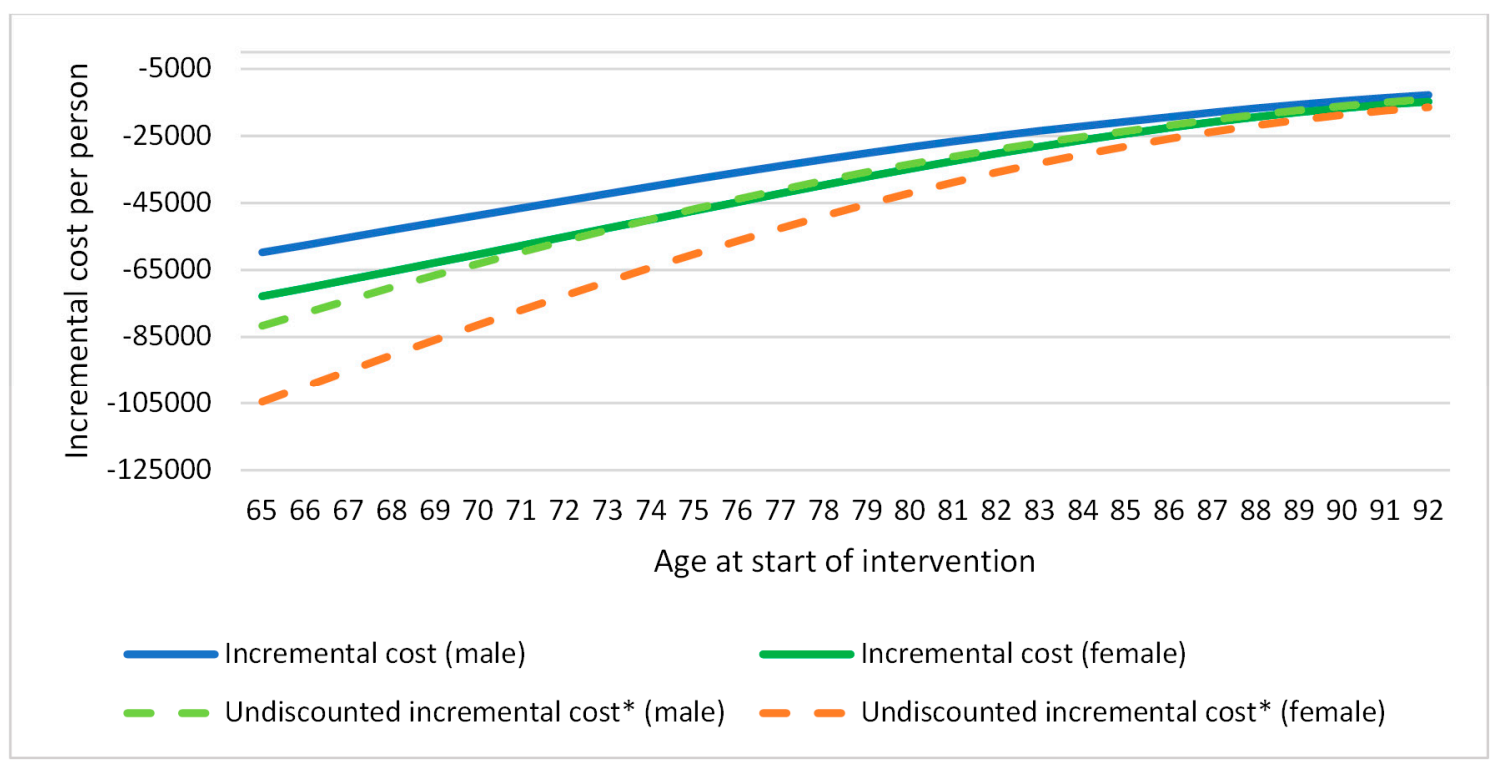

Figure 2. Incremental cost by age (healthcare). Figure 2 presents incremental costs for every age-gender combination in the specified target population. * Undiscounted incremental costs are those not applying the discount factors for costs and effects which are used to estimate outcomes while taking into account the future costs and health effects. This means adjusting for differences in the timing of costs (estimated expenditure) compared to health benefits (outcomes). The discount factor rate applied to this study was $3.5 \%$ for both cost and outcomes.

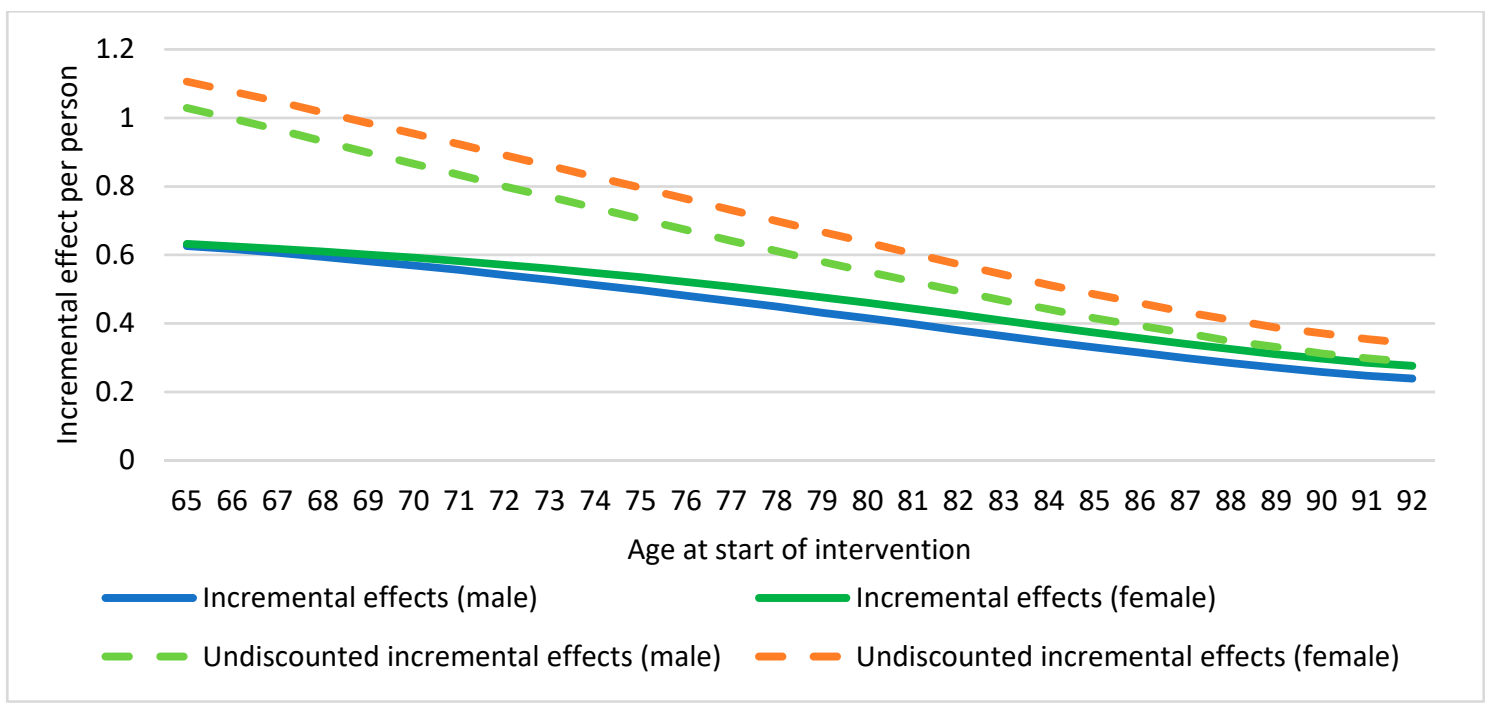

Figure 3. Incremental effects by age. Figure 3 presents incremental effects for every age-gender combination in the specified target population. 
The incremental cost-effectiveness ratio (ICER) represented the overall impact of the physical exercise programme on costs and quality-adjusted life years (QALYs) for the total target population. The cost-effectiveness results are shown in both Table 6 and Figure 4. In this case, the ICER was in the lower-right quadrant (Figure 4). This position of the ICER indicated that the physical exercise programme was cost-effective in comparison to the usual care alternative. The diagonal line represents the willingness to pay (WTP) per additional QALY gained, which is the maximum amount that a patient is willing to give in exchange for a better quality of life.

Table 6. Incremental costs and health-related quality of life (HRQoL) effects (cost-effectiveness).

\begin{tabular}{lc}
\hline Incremental Cost and HRQoL * Effects & Effect Result \\
\hline Incremental cost (healthcare) & $-44,832.92$ \\
Incremental effects & 0.513 \\
Incremental cost-effectiveness ratio (healthcare) & Dominant \\
\hline
\end{tabular}

* HRQoL: health-related quality of life.

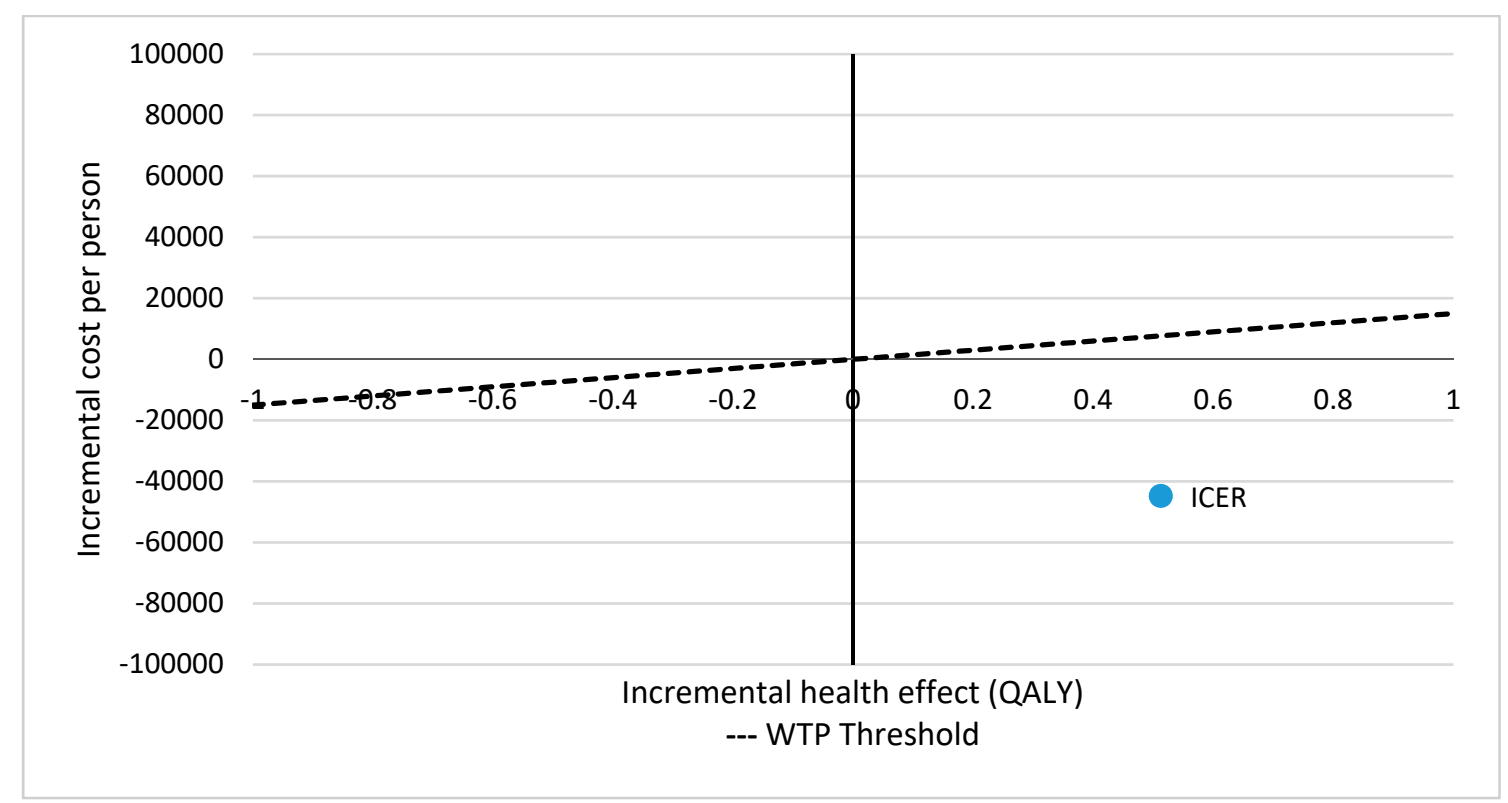

Figure 4. Cost-effectiveness plane (healthcare costs). Figure 4 presents the overall impact of the intervention on the costs and effects of the whole target population ICER: incremental cost-effectiveness ratio; WTP Threshold: willingness to pay (WTP) threshold per quality-adjusted life-year (QALY).

\section{Discussion}

The effectiveness analyses results showed that our intervention significantly reduced the risk of falling by $45.4 \%$ after attending for 9 months the physical exercise programme; while in the same time period the comparison group suffered a slight increase in the risk of falling $(+0.8 \%)$. This reduction is higher than the one showed in other studies. The studies where the OTAGO Programme was validated reported a reduction of falls up to $35 \%$ and the systematic review of 159 studies carried out by Gillespie et al. [2] indicated that interventions based on multicomponent exercise programmes reduced the risk of falling about $30 \%$.

In regard to frailty, our physical exercise programme showed a statistically significant reduction of frailty of $31 \%$ among intervention group participants, while the comparison group experienced a reduction of $4 \%$, which has not been found statistically significant. In the absence of consensus on the operational definition of frailty, the different studies that have tried to understand the effects of an 
exercise programme on frailty assessed frailty based on different criteria [4]. Therefore, the comparison of our results on frailty with the ones found in other studies presents certain difficulties.

Regarding the secondary outcomes, our work showed significant improvement in physical performance, in line with systematic reviews [41-44]. In most of these studies, physical performance was measured by SPPB, the one used in our study. After our intervention, intervention group participants presented improvements in all SPBB three tests, being this improvement only significant for the balance. In contrast, the systematic revision carried out by Giné-Garriga et al. [43] concluded that exercise programmes applied to frail older adults significantly improved the gait speed and chair stands test, while this improvement did not occur in the balance test. In addition, the revision of Cadore et al. [37] also found improvements in gait speed and in balance and Haider et al. [44] found improvements in balance. Perhaps the absence of significant improvement in gait speed among the intervention group participants in this study was due to the fact that our physical exercise programme was mainly focused on balance training and strength training.

As for body composition, which has also been used as a criterion of frailty in some research, our study showed a significant reduction in BMI in the participants of the physical exercise programme. Among the studies that have included body composition as a measure of frailty, the review of Theou et al. [45] found no significant results for this criterion in frail older people after participating in an intervention based on exercise.

Limitations in ADLs have been also included in many of these studies as a criterion of frailty since it has been found as both a risk factor and a consequence of falls [46] and frailty [10,47]. Accordingly, and as expected, after the intervention, the intervention group presented a significant reduction of ADLs limitations. Similar results were found in the systematic revisions of Chou et al. [48] and Theou et al. [45].

In regard to the other health variables related to falls and frailty, the results confirmed that our intervention had effects beyond the intervention variables: the intervention group experienced greater improvements in self-care agency, in self-efficacy related to falls, in physical quality of life and in the average number of doctor visits and hospitalisations. However, these results showed statistically significant improvements only in self-care agency and the average number of doctor visits. As for self-care agency, surprisingly, the comparison group also experienced a significant improvement in this variable. Perhaps the assessment, which includes many questions about health and other health-related variables, raised awareness of health and care among the study participants -including comparison group participants-and thus the assessment itself encouraged them to perform self-care behaviours [49]. On the other hand, the average number of doctor visits was reduced by $33 \%$ and hospitalisation rate by $50 \%$ among participants who received the intervention in comparison with the comparison group who rose by $14 \%$ their hospitalisation rates. Several studies have established the relationship between frailty and hospitalisation, and between falls and emergency room visits and hospitalisation $[4,10,50]$. Therefore, a reduction of risk of falling and/or frailty could lead to a reduction of the use of health resources.

Finally, the cost-effectiveness analyses of the intervention conducted using the MAFEIP tool showed the potential for cost savings that our physical exercise programme provided in comparison to the usual care scenario, which was provided to the comparison group participants. Similar results have been found for other multicomponent programmes and in several systematic reviews and meta-analyses. The OTAGO Programme was cost-saving in terms of falls prevented in people older than 80 [51]. The reviews of Davis et al. [52] and Balzer et al. [53] showed that tailored multicomponent interventions for older adults suffering from risk of falling, that include balance and strength training, were cost-effective or cost-saving. Recently, the review of Apostolo et al. [22] examined the effectiveness and cost-effectiveness of interventions for frailty in older adults, concluding that physical exercise interventions showed to be generally effective for reducing frailty but only when conducted in groups. The economic estimation demonstrated that this type of intervention, compared to usual care, can provide better value for money. 
The results of the present study should be interpreted in the context of potential limitations. First, the lack of randomisation in the distribution of participants in the intervention and comparison groups. Aware of such limitations, this study tested the homogeneity between groups by analysing the equivalence between the initial sample of subjects assigned to the intervention group and the comparison group. Another limitation of this research is the sample size, since this was reduced to 55 participants in the intervention group and 136 participants in the comparison group, which increased risk of type I error. However, it is important to note that the achieved sample size is considered appropriated in research [54] and the alpha value of the primary outcomes was $\leq 0.01$, which reduces the probability of type I error. Nevertheless, the study results should be interpreted with caution and it is recommended to replicate the study with a deeper analysis of other relevant variables which could explain partially the positive effect of the intervention (motivation, socioeconomic variables, etc.).

\section{Conclusions}

In conclusion, the results of this study highlight the need to implement multicomponent physical exercise programmes to reduce the risk of falling and frailty, two of the most prevalent conditions which have a greater impact on older adults' health. In this regard, our novel 9-month multicomponent physical exercise programme that offers a modified format of OTAGO programme including evidence-based improvements has demonstrated to be both potentially effective and less costly than usual care.

Supplementary Materials: The following are available online at http://www.mdpi.com/1660-4601/16/12/2086/s1, S1: Multicomponent physical exercise programme.

Author Contributions: T.A.-B. and E.D.-F. contributed to the conception and design of the project; T.A.-B. contributed to the data acquisition; T.A.-B., E.D.-F. and M.F.-G. contributed to the analysis and interpretation of the data; T.A.-B. drafted the article. All authors supplied critical revisions to the manuscript and gave final approval of the version to be published.

Funding: This work was conducted in the frame of a research project (Urban Health Centre 2.0: Integrated health and social care pathways, early detection of frailty, management of polypharmacy and prevention of falls for active and healthy ageing in European cities-UHCE) supported by supported by the European Union, CHAFEA, Third Health Programme, grant number 20131201. The UHCE study was registered in the ISRCTN (International Standard Randomised Controlled Trial Number) registry under number ISRCTN52788952.

Acknowledgments: We would like to thank all participating older persons and all organisations and professionals involved in this study.

Conflicts of Interest: The authors declare that they have no competing interests.

\section{References}

1. Ong, A.D.; Uchino, B.N.; Wethington, E. Loneliness and Health in Older Adults: A Mini-Review and Synthesis. Gerontology 2016, 62, 443-449. [CrossRef] [PubMed]

2. Gillespie, L.D.; Robertson, M.C.; Gillespie, W.J.; Sherrington, C.; Gates, S.; Clemson, L.M.; Lamb, S.E. Interventions for preventing falls in older people living in the community. Cochrane Database Syst. Rev. 2012, 9, CD007146. [CrossRef] [PubMed]

3. European Commission. Injury Database. European Commission, 2016. Available online: http://ec.europa.eu/ health/data_collection/databases/idb/index_en.htm (accessed on 2 May 2018).

4. Fried, L.P.; Tangen, C.M.; Walston, J.; Newman, A.B.; Hirsch, C.; Gottdiener, J.; Seeman, T.; Tracy, R.; Kop, W.J.; Burke, G.; et al. Frailty in older adults: Evidence for a phenotype. J. Gerontol. Ser. A Biol. Sci. Med. Sci. 2001, 56, 146-149. [CrossRef] [PubMed]

5. Gobbens, R.J.; Luijkx, K.G.; Wijnen-Sponselee, M.T.; Schols, J.M. Toward a conceptual definition of frail community dwelling older people. Nurs. Outlook 2010, 58, 76-86. [CrossRef] [PubMed]

6. Gobbens, R.J.; van Assen, M.A.; Luijkx, K.G.; Wijnen-Sponselee, M.T.; Schols, J.M. The Tilburg Frailty Indicator: Psychometric properties. J. Am. Med. Dir. Assoc. 2010, 11, 344-355. [CrossRef] [PubMed]

7. Markle-Reid, M.; Browne, G. Conceptualizations of frailty in relation to older adults. J. Adv. Nurs. 2003, 44, 58-68. [CrossRef] [PubMed] 
8. Coelho, T.; Paul, C.; Gobbens, R.; Fernandes, L. Frailty as a predictor of short-term adverse outcomes. PeerJ 2015, 3, 1121. [CrossRef] [PubMed]

9. Morley, J.E.; Vellas, B.; van Kan, G.A.; Anker, S.D.; Bauer, J.M.; Bernabei, R.; Cesari, M.; Chumlea, W.C.; Doehner, W.; Evans, J.; et al. Frailty consensus: A call to action. J. Am. Med. Dir. Assoc. 2013, 14, 392-397. [CrossRef]

10. Romero, L.; Abizanda, P. Fragilidad como predictor de episodios adversos en estudios epidemiológicos: Revisión de la literatura. Rev. Esp. Geriatr. Gerontol. 2013, 48, 285-289. [CrossRef]

11. World Health Organization. WHO Global Report on Falls Prevention in Older Age; World Health Organization: Geneva, Switzerland, 2007; Available online: http://www.who.int/entity/ageing/publications/ Falls_prevention7March.pdf (accessed on 2 May 2018).

12. World Health Organization. WHO Global Report on Falls Prevention in Older Age; World Health Organization: Geneva, Switzerland, 2012; Available online: http://www.who.int/ageing/publications/ Falls_prevention7March.pdf?ua=1 (accessed on 2 May 2018).

13. Bock, J.O.; König, H.H.; Brenner, H.; Haefeli, W.E.; Quinzler, R.; Matschinger, H.; Saum, K.U.; Schöttker, B.; Heider, D. Associations of frailty with health care costs-Results of the ESTHER cohort study. BMC Health Serv. Res. 2016, 16, 128. [CrossRef]

14. Florence, C.S.; Bergen, G.; Atherly, A.; Burns, R.; Stevens, J.; Drake, C. Medical Costs of Fatal and Nonfatal Falls in Older Adults. J. Am. Geriatr. Soc. 2018, 66, 693-698. [CrossRef] [PubMed]

15. Casas Herrero, A.; Izquierdo, M. Physical exercise as an efficient intervention in frail elderly persons. An. Sist. Sanit. Navar. 2012, 35, 69-85. [CrossRef] [PubMed]

16. El-Khoury, F.; Cassou, B.; Charles, M.A.; Dargent-Molina, P. The effect of fall prevention exercise programmes on fall induced injuries in community dwelling older adults: Systematic review and meta-analysis of randomised controlled trials. BMJ 2013, 347, f6234. [PubMed]

17. Sherrington, C.; Whitney, J.C.; Lord, S.R.; Herbert, R.D.; Cumming, R.G.; Close, J.C. Effective exercise for the prevention of falls: A systematic review and meta-analysis. J. Am. Geriatr. Soc. 2008, 56, 2234-2243. [CrossRef] [PubMed]

18. Sherrington, C.; Tiedemann, A.; Fairhall, N.; Close, J.C.T.; Lord, S.R. Exercise to prevent falls in older adults: An updated meta-analysis and best practice recommendations. N. S. W. Public Health Bull. 2011, 22, 78-83. [CrossRef]

19. Campbell, A.J.; Robertson, M.C. The Otago Exercise Programme to Prevent Falls in Older: A Homebased; Individually Tailored Strength and Balance Retraining Programme; Otago Medical School, University of Otago: Dunedin, New Zealand, 2003.

20. Stevens, J.A.; Burns, E. A CDC Compendium of Effective Fall Interventions: What Works for Community-Dwelling Older Adults; National Center for Injury Prevention and Control of the Centers for Disease Control and Prevention: Atlanta, GA, USA, 2015.

21. Thomas, S.; Mackintosh, S.; Halbert, J. Does the 'Otago exercise programmeme' reduce mortality and falls in older adults?: A systematic review and metaanalysis. Age Ageing 2010, 39, 681-687. [CrossRef]

22. Apóstolo, J.; Cooke, R.; Bobrowicz-Campos, E.; Santana, S.; Marcucci, M.; Cano, A.; Vollenbroek-Hutten, M.; Germini, F.; D'Avanzo, B.; Gwyther, H.; et al. Effectiveness of interventions to prevent pre-frailty and frailty progression in older adults: A systematic review. JBI Database Syst. Rev. Implement. Rep. 2018, 16, 140-232. [CrossRef]

23. Kyrdalen, I.L.; Moen, K.; Roysland, A.S.; Helbostad, J.L. The Otago exercise programme performed as group training versus home training in fall-prone older people: A randomized controlled trial. Physiother. Res. Int. 2014, 92, 108-116. [CrossRef]

24. American Geriatrics Society, British Geriatrics Society. AGS/BGS Clinical Practice Guideline: Prevention of Falls in Older Persons; American Geriatrics Society: New York, NY, USA, 2010.

25. Curcio, C.L.; Gómez, F. Temor a caer en ancianos: Controversias en torno a un concepto y a su medición. Hacia la Promoción de la Salud 2012, 17, 186-204.

26. Ambrose, A.F.; Paul, G.; Hausdorffb, J.M. Risk factors for falls among older adults: A review of the literature. Maturitas 2013, 75, 51-61. [CrossRef]

27. NICE. Falls: Assessment and Prevention of Falls in Older People; Clinical Guideline; No. 161; National Institute for Health and Care Excellence (NICE): London, UK, 2013. 
28. Gobbens, R.J.; Schols, J.M.; van Assen, M.A. Exploring the efficiency of the Tilburg Frailty Indicator: A review. Clin. Interv. Aging 2017, 12, 1739-1752. [CrossRef] [PubMed]

29. Suurmeijer, T.P.B.M.; Kempen, G.I.J.M. Behavioural changes as an outcome of disease: The development of an instrument. Int. J. Health Sci. 1990, 1, 189-194.

30. Orem, D.E. Nursing: Concepts of Practice, 5th ed.; Mosby: Saint Louis, MO, USA, 1995.

31. Alhambra-Borrás, T.; Durá-Ferrandis, E.; Garcés-Ferrer, J.; Sánchez-García, J. The Appraisal of Self-care Agency scale Revised (ASAR): Adaptation and validation in a Spanish seniors' sample. Span. J. Psychol. 2017, 20, e48. [CrossRef] [PubMed]

32. Yardley, L.; Beyer, N.; Hauer, K.; Kempen, G.; Piot-Ziegler, C.; Todd, C. Development and initial validation of the Falls Efficacy Scale-International (FES-I). Age Ageing 2005, 34, 614-619. [CrossRef] [PubMed]

33. Tinetti, M.E.; Richman, D.; Powell, L. Falls efficacy as a measure of fear of falling. J. Gerontol. 1990, 45, 239-243. [CrossRef]

34. Litwin, H.; Erlich, B.; Dunsky, A. The Complex Association between Fear of Falling and Mobility Limitation in Relation to Late-Life Falls: A SHARE-Based Analysis. J. Aging Health 2018, 30, 987-1008. [CrossRef] [PubMed]

35. Ware, J.E.; Kosinsiki, M.; Keller, S.D. A 12-item short-form health survey: Construction of scales and preliminary tests of reliability and validity. Med. Care 1996, 34, 220-233. [CrossRef] [PubMed]

36. Guralnik, J.M.; Simonsick, E.M.; Ferrucci, L.; Glynn, R.J.; Berkman, L.F.; Blazer, D.G.; Scherr, P.A.; Wallace, R.B. A short physical performance battery assessing lower extremity function: Association with self-reported disability and prediction of mortality and nursing home admission. J. Gerontol. 1994, 49, 85-94. [CrossRef]

37. Cadore, E.L.; Rodríguez-Mañas, L.; Sinclair, A.; Izquierdo, M. Effects of different exercise interventions on risk of falls, gait ability, and balance in physically frail older adults: A systematic review. Rejuvenation Res. 2013, 16, 105-114. [CrossRef] [PubMed]

38. Matsuda, P.N.; Shumway-Cook, A.; Ciol, M.A. The effects of a home-based exercise programme on physical function in frail older adults. J. Geriatr. Phys. Ther. 2010, 33, 78-84. [PubMed]

39. Boehler, C.E.; de Graaf, G.; Steuten, L.; Yang, Y.; Abadie, F. Development of a Web-based Tool for the Assessment of Health and Economic Outcomes of the European Innovation Partnership on Active and Healthy Ageing (EIP on AHA). BMC Med. Inform. Decis. Mak. 2015, 3, S4. [CrossRef] [PubMed]

40. Gray, A.; Rivero-Arias, O.; Clarke, P. Estimating the association between SF-12 responses and EQ-5D utility values by response mapping. Med. Decis. Mak. 2006, 26, 18-29. [CrossRef] [PubMed]

41. Daniels, R.; van Rossum, E.; de Witte, L.; Kempen, G.I.; van den Heuvel, W. Interventions to prevent disability in frail community-dwelling elderly: A systematic review. BMC Health Serv. Res. 2008, 8, 278. [CrossRef] [PubMed]

42. de Vries, N.M.; van Ravensbergb, C.D.; Hobbelenb, J.S.M.; Olde Rikkertd, M.G.M.; Staal, J.B.; Nijhuis-van der Sandena, M.W.G. Effects of physical exercise therapy on mobility, physical functioning, physical activity and quality of life in community-dwelling older adults with impaired mobility, physical disability and/or multi-morbidity: A meta-analysis. Ageing Res. Rev. 2012, 11, 136-149. [CrossRef] [PubMed]

43. Giné-Garriga, M.; Roqué-Fíguls, M.; Coll-Planas, L.; Sitja-Rabert, M.; Salva, A. Physical exercise interventions for improving performance-based measures of physical function in community-dwelling, frail older adults: A systematic review and meta-analysis. Arch. Phys. Med. Rehabil. 2014, 95, 753-769. [CrossRef] [PubMed]

44. Haider, S.; Luger, E.; Kapan, A.; Titze, S.; Lackinger, C.; Schindler, K.E.; Dorner, T.E. Associations between daily physical activity, handgrip strength, muscle mass, physical performance and quality of life in prefrail and frail community-dwelling older adults. Qual. Life Res. 2016, 25, 3129-3138. [CrossRef] [PubMed]

45. Theou, O.; Stathokostas, L.; Roland, K.P.; Jakobi, J.M.; Patterson, C.; Vandervoort, A.A.; Jones, G.R. The effectiveness of exercise interventions for the management of frailty: A systematic review. J. Aging Res. 2011, 2011, 569194. [CrossRef] [PubMed]

46. Abizanda, P.; Gómez-Pavón, J.; Martín-Lesende, I.; Baztán, J.J. Detección y prevención de la fragilidad: Una nueva perspectiva de prevención de la dependencia en las personas mayores. Med. Clín. 2010, 135, 713-719. [CrossRef] [PubMed]

47. Ávila-Funes, J.A.; Helmer, C.; Amieva, H.; Barberger-Gateau, P.; Le Goff, M.; Ritchie, K.; Portet, F.; Carrière, I.; Tavernier, B.; Gutiérrez-Robledo, L.M.; et al. Frailty among community-dwelling elderly people in France: The three-city study. J. Gerontol. A Biol. Sci. Med. Sci. 2008, 63, 1089-1096. [CrossRef] [PubMed] 
48. Chou, C.H.; Hwang, C.L.; Wu, Y.T. Effect of exercise on physical function, daily living activities, and quality of life in the frail older adults: A meta-analysis. Arch. Phys. Med. Rehabil. 2012, 93, 237-244. [CrossRef]

49. Eckman, M. Enfermería Geriátrica; Manual Moderno: Mexico DF, Mexico, 2012.

50. Kojima, G. Frailty as a predictor of hospitalisation among community-dwelling older people: A systematic review and meta-analysis. J. Epidemiol. Community Health 2016, 70, 722-729. [CrossRef] [PubMed]

51. Robertson, M.C.; Devlin, N.; Gardner, M.M.; Campbell, A.J. Effectiveness and economic evaluation of a nurse delivered home exercise programme to prevent falls. 1: Randomised controlled trial. BMJ 2001, 322, 697. [CrossRef] [PubMed]

52. Davis, J.C.; Robertson, M.C.; Ashe, M.C.; Liu-Ambrose, T.; Khan, K.M.; Marra, C.A. Does a home based strength and balance programme in people aged $\geq 80$ years provide the best value for money to prevent falls? A systematic review of economic analyses of falls prevention interventions. Br. J. Sports Med. 2010, 44, 80-89. [CrossRef] [PubMed]

53. Balzer, K.; Bremer, M.; Schramm, S.; Lühmann, D.; Raspe, H. Falls prevention for the elderly. GMS Health Technol. Assess. 2012, 8. [CrossRef]

54. Hair, J.F.; Anderson, R.E.; Tathan, R.L.; Black, W.C. Multivariate Data Analysis, 5th ed.; Pearson: London, UK, 1999.

(C) 2019 by the authors. Licensee MDPI, Basel, Switzerland. This article is an open access article distributed under the terms and conditions of the Creative Commons Attribution (CC BY) license (http://creativecommons.org/licenses/by/4.0/). 